\title{
Avaliação dos fatores de risco laborais e físicos para doenças cardiovasculares em motoristas de transporte urbano de ônibus em Montes Claros (MG)
}

\author{
Evaluation of labor-related and physical risk factors \\ for cardiovascular disease in drivers of urban transport buses \\ in Montes Claros in the state of Minas Gerais
}

Andréia Farias Alquimim ${ }^{1}$

Ana Beatris Cezar Rodrigues Barral ${ }^{1}$

Kênnya Caroline Gomes ${ }^{1}$

Mayra Costa de Rezende ${ }^{1}$
${ }^{1}$ Faculdades Integradas Pitágoras. Avenida Mairtina, Ibituruna. 39400-000 Montes Claros MG. andreiaalquimim@hotmail.com
Abstract The scope of this study was to evaluate risk factors for cardiovascular disease among bus drivers in Montes Claros in the state of Minas Gerais. A semi-structured questionnaire covering personal, anthropometric, professional and labor-related data was used, in addition to a questionnaire on the level of stress. 53 bus drivers were surveyed and the average age was 30 to 39 years of age. $81.1 \%$ were non-smokers; $58 \%$ of the sample were teetotalers; and 50\% took regular exercise. In the assessment of BMI, 40 drivers (75.4\%) were overweight. The prevalence in eating habits revealed excess consumption of sugar (66.0\%), fat (64.2\%), coffee (69.8\%), salt (60.4\%), coca cola (64.2\%) and soft drinks (54.7\%). Among reports of chronic diseases, no diabetic (98.1\%) or hypertensive (94.3\%) drivers were observed. Most of the sample (69.7\%) had normal stress levels. With respect to laboratory data, the vast majority of drivers had hypertriglyceridemia and hypercholesterolemia. HDL levels were satisfactory, and the LDL revealed normal and desirable levels in more than half of the sample. The prevalence of cardiovascular disease was low.

Key words Risk factors, Cardiovascular disease, Bus driver, Urban transport
Resumo Este estudo buscou avaliar os fatores de risco para doenças cardiovasculares em motoristas de ônibus em Montes Claros (MG). Foi empregado um questionário semiestruturado abordando dados pessoais, antropométricos, profissionais e laborais; e outro referente ao grau de estresse. Foram pesquisados 53 motoristas de ônibus e a média de idade foi de 30 a 39 anos, e dessa população, 81,1\% diziam não ser fumantes, 58\% não consumiam bebida alcoólica e 50\% praticavam algum tipo de exercício. Na avaliação do IMC 40 motoristas $(75,4 \%)$ estavam com excesso de peso. A prevalência dos hábitos alimentares foi de excesso consumo de açúcar (66,0\%), de gordura (64,2\%), de café (69,8\%), de sal (60,4\%), de Coca Cola (64,2\%) e de refrigerante (54,7\%). Dentre os relatos de doenças crônicas não foram observados motoristas diabéticos (98,1\%) e nem hipertensos (94,3\%). A maioria da amostragem, 69,7\% teve nível de estresse normal. Em relação aos dados laboratoriais, a grande maioria dos motoristas apresentou hipertrigliceridemia e hipercolesterolemia. Os niveis de HDL estavam satisfatórios, e o de LDL apresentou nível normal e desejável em mais da metade da amostra. A prevalência para doença cardiovascular mostrou-se baixa.

Palavras-chave Fatores de risco, Doença cardiovascular, Motorista de ônibus, Transporte urbano 


\section{Introdução}

O transporte coletivo tem uma função urbana e social que interage diretamente com o desenvolvimento e a organização das cidades. A forma de ocupação espacial urbana define uma determinada rede de transporte, conforme investimento na infraestrutura viária, merecendo destaque por esse motivo ${ }^{1}$.

O transporte público urbano é responsável por cerca de $70 \%$ dos deslocamentos mecanizados nas cidades brasileiras, sendo o ônibus o meio quase que exclusivamente responsável pela realização dessa movimentação. Diante disso, o motorista de ônibus torna-se um profissional muito importante, sendo o responsável direto pelo patrimônio (ônibus), pela integridade física dos passageiros e pela própria imagem da empresa ${ }^{2}$.

Diferente de outros profissionais, que desempenham suas funções em ambientes fechados, climatizados e relativamente confortáveis, o motorista de ônibus o faz em ambiente público: o trânsito. Não possui, portanto, um local restrito e bem definido para realizar suas tarefas e, ao contrário, trabalha fora dos portões da empresa, estando sujeito a intempéries, como o clima, o trajeto das vias, as condições do tráfego, a falha nos equipamentos e a pressão para cumprir horário ${ }^{3}$.

O motorista de ônibus está sujeito a um grande número de situações com potencial estressor, contribuindo para isso a duração da jornada de trabalho, a situação precária do asfalto, os baixos salários, o estado dos veículos, os ruídos, a temperatura, o congestionamento, a relação com o público e a insegurança (exposto a assalto). Tais fatores favorecem o aparecimento de doenças ocupacionais ${ }^{4}$.

Além disso, durante o cotidiano, o motorista expõe seu corpo a muitas agressões, como o sedentarismo e a alimentação inadequada, os quais constituem importantes fatores de risco para doenças cardiovasculares. Por esses motivos, é importante que a empresa não o veja apenas sob o prisma do cumprimento de exigências ou de interesses econômicos, mas sim como seres singulares inseridos na organização do trabalho ${ }^{4}$.

O crescimento da frota de veículos, sem um concomitante aumento do sistema viário e um bom planejamento urbano, tem sido responsável pelo aumento do percurso e, consequentemente, pela crescente agressividade dos motoristas causada por distúrbios emocionais. A sensação de perda de liberdade que os cidadãos podem sofrer gera distúrbios de comportamento, com consequências imprevisíveis. Somando-se a isso, as preocupações diárias e os horários a serem cumpridos conduzem o motorista, em especial, à irritabilidade e à agressividade. Além de tornarem-se um risco potencial aos acidentes de trânsito, esses fatores também podem gerar consequências nocivas à saúde, como por exemplo, o estresse. O congestionamento do tráfego se relaciona, ainda, com alta incidência de taquicardia, epigastralgia e problemas cardíacos decorrentes de descarga adrenérgica ${ }^{5}$.

A incidência de infarto do miocárdio entre o grupo de motoristas de ônibus atingiu níveis superiores a de outras doenças nesse mesmo grupo, sendo o risco maior nos que trabalham na área urbana ${ }^{6}$.

Em função do exposto e pela inexistência de estudos representativos da população de motoristas de ônibus em Montes Claros, buscou-se preencher essa lacuna, assim, o presente estudo foi conduzido com o objetivo de se avaliar os fatores de risco relacionados às atividades de trabalho e pessoal para doenças cardiovasculares em motoristas de transporte urbano de ônibus em Montes Claros (MG).

\section{Metodologia}

O estudo conduziu a uma investigação de caráter quantitativo, transversal, observacional e descritivo.

O público alvo do presente trabalho foi composto pelos motoristas de transporte urbano de ônibus em Montes Claros, pertencentes ao sexo masculino, que trabalham no turno diurno, semanalmente, e que executam essa função na empresa de transporte coletivo da cidade, há pelo menos um ano.

Para a coleta de dados foi empregado um questionário semiestruturado abordando dados pessoais, antropométricos, profissionais e laborais. Após esta etapa, os motoristas foram submetidos a uma avaliação antropométrica com peso e altura para cálculo de índice de massa corpórea, que é obtido pela divisão do peso pela altura ao quadrado. Os motoristas foram classificados, segundo os critérios estabelecidos pelas "Diretrizes para Cardiologistas sobre Excesso de Peso e Doença Cardiovascular”, como: saudável (IMC menor que $25 \mathrm{~kg} / \mathrm{m} 2$ ), sobrepeso (IMC entre 25 e $29,9 \mathrm{~kg} / \mathrm{m} 2$ ) e obesos (IMC maior ou igual a $30 \mathrm{~kg} / \mathrm{m} 2$ ).

A medida da circunferência abdominal obedeceu aos parâmetros impostos pela V Diretrizes Brasileira de Hipertensão Arterial, sendo classificado como ideal a menor ou igual a 94 centí- 
metros (cm), como de risco aumentado a de 95 a $101 \mathrm{~cm}$ e de risco muito aumentado quando maior ou igual a $102 \mathrm{~cm}$. Ainda, conforme a Diretriz citada, o motorista é considerado hipertenso quando a pressão sistólica for maior ou igual a $140 \mathrm{mmHg}$ e a diastólica, maior ou igual a $90 \mathrm{mmHg}$.

Para a classificação do perfil lipídico, foram considerados os valores de referência limítrofes da III Diretrizes Brasileira sobre Dislipidemias para indivíduos maiores de 20 anos: colesterol total de 200-239 mg/dl, LDLC de 130-139 mg/dl e TG de 150-200 mg/dl. Quanto ao nível de HDLC, como valor alto se for maior que $60 \mathrm{mg} / \mathrm{dl}$.

$\mathrm{Na}$ coleta dos dados antropométricos, foram utilizados: balança de precisão Welmy, calculadora, fita métrica de dois metros. Para aferir a pressão arterial empregaram-se esfigmomanômetro e estetoscópio, ambos da marca BD. Na coleta de sangue, os motoristas tiveram que permanecer em jejum por doze horas até o momento da punção venosa. Agulhas descartáveis foram utilizadas.

Após a coleta dos dados, eles foram tabulados e analisados por meio de métodos descritivos e estatísticos. Para isso, usou-se a porcentagem, odds ration, " $t$ " student e p com significância quando menor ou igual a 0,05 .

\section{Discussão}

Foram pesquisados 53 motoristas de ônibus e, de acordo com a Tabela 1 , a média de idade foi de 30 a 39 anos (74,5\%), sendo a maioria casado/ união estável/morando junto. No estudo de Battiston et al. ${ }^{3}$ numa amostra composta por 21 motoristas de ônibus urbano, a distribuição dos participantes nas faixas etárias foi de $47,6 \%$ para motoristas de 25 a 40 anos e de $52,4 \%$ para os de 41 a 56 anos, sendo todos casados.

O mesmo ocorreu no estudo de Landim e Guimarães ${ }^{7}$, já que a amostra apresentou-se jovem, com a maior parte na faixa dos 36 aos 50 anos.

Alguns motoristas apresentaram estilo de vida saudável, sendo que $(81,1 \%)$ da população diziam não ser fumantes.

Reafirmando os dados do presente estudo, Landim e Guimarães ${ }^{7}$ afirmam que, dos 122 motoristas de transporte coletivo urbano de Teresina, no Piauí, a prevalência do hábito de fumar foi pequena, em torno de $20 \%$ da amostra pesquisada. Isto pode sugerir que as campanhas de combate ao tabagismo e a legislação atual no Brasil, desenvolvidas pelas autoridades governamentais e judiciais, com participação da sociedade como um todo, vêm surtindo o efeito desejado.

Quanto à bebida alcoólica 58\% da amostra não consumiam e em relação à atividade física $50 \%$ praticavam algum tipo de exercício. $\mathrm{Na}$ avaliação do IMC, no entanto, 40 motoristas $(75,4 \%)$ estavam com excesso de peso (Tabela 1 ).

Conforme Rocha et al. ${ }^{8}$ na investigação de 133 motoristas de transporte coletivo de Fortaleza, Ceará, em relação à ingestão de bebidas alcoólicas, 63,9\% deles bebiam e $76(57,1 \%)$ referiram praticar exercícios físicos, contradizendo os dados numéricos encontrados na pesquisa atual. No entanto, em relação à obesidade há similaridade, visto que $63(47,4 \%)$ estavam com sobrepeso (pré-obesidade) e $50(37,7)$ estavam na faixa de risco aumentado em relação à circunferência abdominal ${ }^{8}$.

No estudo de Costa et al. ${ }^{9}$ com motoristas de ônibus de SP, foi demonstrado que tanto os que praticam quanto os que não praticam atividade ou esporte não demonstraram significância estatística em relação ao sobrepeso/obesidade, por outro lado, em Belo Horizonte a ausência de esportes aumentou em $27,6 \%$ esse risco para os motoristas.

Em relação a condições relacionada ao trabalho, o tempo médio de trabalho na empresa dos funcionários entrevistados foi acima de 5 anos $(37,7 \%)$ e 40 motoristas realizavam pausa durante o itinerário. Desses, 32 (84,3\%) faziam mais de 2 pausas (Tabela 1 ).

Consoante a Costa et al. ${ }^{9}$ aproximadamente $42,86 \%$, do total de 306 dos pesquisados em São Paulo e Belo Horizonte, possuem menos de três anos de experiência na função como motorista e $57,14 \%$ trabalham há mais de três anos na empresa, corroborando também com o tempo de trabalho na empresa da amostra da pesquisa.

Ainda no estudo de Costa et al. ${ }^{9}$ no que se refere ao número de pausa, em 1 hora de intervalo, $68,75 \%$ dizem permanecer na sala para os motoristas no próprio terminal. Nesse momento os motoristas realizam suas refeições e outras atividades como a ida ao banheiro ou o próprio descanso.

Por outro lado, 31,25\% possuem uma pausa maior, porém sua jornada de trabalho se estende a outro turno. Alguns motoristas retornam as suas casas e outros permanecem nos próprios terminais ${ }^{9}$. Isso não foi observado no presente estudo, já que os motoristas não possuem uma sala onde possam fazer suas refeições e descansar, além de suas pausas serem de no máximo cinco minutos.

A prevalência dos hábitos alimentares foi de excesso consumo de açúcar $(66,0 \%)$, gordura 
$(64,2 \%)$, café $(69,8 \%)$, sal $(60,4 \%)$, coca cola $(64,2 \%)$ e de refrigerante $(54,7 \%$.) (Tabela 1$)$.

Considerando que uma alimentação saudável deve evitar o consumo elevado de gorduras, nota-se que os motoristas têm o hábito de inge- rir alimentos ricos em gordura com frequência. Pois, $40 \%$ referem ingerir frituras com frequência e $30,9 \%$ consumir de quatro a seis vezes na semana lanches (salgadinhos, pizzas, cachorro quente, sanduíches, etc). Quanto ao consumo de

Tabela 1. Distribuição dos motoristas da amostra segundo variáveis investigadas.

\begin{tabular}{|c|c|c|c|c|c|}
\hline Variáveis & n & $\%$ & Variáveis & $\mathbf{n}$ & $\%$ \\
\hline Aspectos sociodemográficos & & & Autorrelato de doenças crônicas & & \\
\hline Faixa etária & & & Diabetes & & \\
\hline Abaixo de 30 anos & 12 & 22,6 & Sim & 01 & 1,9 \\
\hline 30 a 39 anos & 20 & 37,7 & Não & 52 & 98,1 \\
\hline Acima de 39 anos & 21 & 39,7 & Hipertensão & & \\
\hline Estado civil & & & Sim & 03 & 5,7 \\
\hline Casado(a)/mora junto/união estável & 38 & 74,5 & Não & 50 & 94,3 \\
\hline Solteiro/separado/divorciado & 13 & 25,5 & História familiar & & \\
\hline Estilo de vida & & & De diabetes & & \\
\hline Consumo de tabaco & & & Sim & 17 & 32,1 \\
\hline Não fumante & 43 & 81,1 & Não & 36 & 67,9 \\
\hline Fumante & 10 & 19,9 & De hipertensão & & \\
\hline Consumo de bebidas alcoólicas & & & Sim & 26 & 49,1 \\
\hline Não & 29 & 58,0 & Não & 27 & 50,9 \\
\hline Sim & 21 & 42,0 & De obesidade & & \\
\hline Prática de Atividade Física & & & Sim & 2 & 3,8 \\
\hline Sim & 24 & 50,0 & Não & 51 & 96,2 \\
\hline Não & 24 & 50,0 & De insuficiência cardíaca & & \\
\hline $\operatorname{Imc}$ & & & Sim & 4 & 7,5 \\
\hline Peso normal & 13 & 24,5 & Não & 49 & 92,5 \\
\hline Excesso de peso & 27 & 50,9 & De acidente vascular & & \\
\hline Obesidade & 13 & 24,5 & Sim & 12 & 22,6 \\
\hline Condições relacionadas ao trabalho & & & Não & 41 & 77,4 \\
\hline Tempo de trabalho na empresa & & & De infarto agudo miocárdio & & \\
\hline 1 ano & 15 & 28,3 & Sim & 12 & 22,6 \\
\hline 2 a 5 anos & 18 & 34,0 & Não & 41 & 77,4 \\
\hline Acima de 5 anos & 20 & 37,7 & Dados laboratoriais & & \\
\hline Faz pausa durante o itinerário & & & Colesterol & & \\
\hline Sim & 40 & 75,5 & Normal & 23 & 43,4 \\
\hline Não & 13 & 24,5 & Limiar elevado & 18 & 34,0 \\
\hline Quantidade de pausas & & & Elevado & 12 & 22,6 \\
\hline 1 pausa & 6 & 15,8 & Trigliceris & & \\
\hline 2 pausas & 19 & 50,0 & Desejável & 17 & 32,1 \\
\hline 3 pausas & 5 & 13,2 & Limiar alto & 11 & 20,8 \\
\hline Mais de 3 pausas & 8 & 21,1 & Elevado & 22 & 41,5 \\
\hline Hábitos alimentares & & & Muito levado & 3 & 5,7 \\
\hline Consumo excessivo de açúcar & & & HDL & & \\
\hline Sim & 35 & 66,0 & Baixo & 33 & 62,3 \\
\hline Não & 18 & 34,0 & Aceitável & 5 & 9,4 \\
\hline Consumo excessivo de gordura & & & Alto & 15 & 28,3 \\
\hline Sim & 19 & 35,8 & LDL & & \\
\hline Não & 34 & 64,2 & Ótimo & 16 & 30,2 \\
\hline Consumo excessivo de café & & & Desejável & 14 & 26,4 \\
\hline Sim & 37 & 69,8 & Limiar alto & 5 & 9,4 \\
\hline Não & 16 & 30,2 & Limiar elevado & 10 & 18,9 \\
\hline Consumo excessivo de sal & & & Muito elevado & 8 & 15,1 \\
\hline Sim & 32 & 60,4 & VLDL & & \\
\hline Não & 21 & 39,6 & Normal & 21 & 39,6 \\
\hline Consumo excessivo de coca-cola & & & Anormal & 32 & 60,4 \\
\hline Sim & 19 & 35,8 & Estresse & & \\
\hline Não & 34 & 64,2 & Nível de estresse & & \\
\hline Consumo excessivo de refrigerante & & & Nível de estresse normal & 36 & 67,9 \\
\hline Sim & 24 & 45,3 & Nível de estresse alterado & 17 & 32,1 \\
\hline Não & 29 & 54,7 & Total & 53 & 100,0 \\
\hline
\end{tabular}


carboidratos simples, $47,3 \%$ referiram consumir diariamente açúcar refinado e 32,7\% consomem de duas a três vezes na semana refrigerante ${ }^{10}$.

Para Lima et al. ${ }^{11}$ o padrão alimentar dos brasileiros tem apresentado mudanças devido ao maior consumo de alimentos industrializados em substituição às tradicionais comidas caseiras. Essas mudanças causadas pelo estilo de vida moderno levam ao consumo excessivo de produtos gordurosos, açúcares, doces e bebidas açucaradas e à diminuição da ingestão de cereais e/ou produtos integrais, frutas e verduras, que são fontes de fibras.

A maioria dos motoristas referiu que após ingressar na profissão, houve aumento de consumo de lanches, como salgadinhos, coxinha, pastel, sendo estes realizados nos terminais de ônibus, sendo tal achado similar ao do estudo de Faria et al. ${ }^{10}$.

Dentre os relatos de doenças crônicas não foram observados motoristas diabéticos $(98,1 \%)$ e nem hipertensos $(94,3 \%)$ (Tabela 1$)$.

Os números aqui revelados surpreendem, de algum modo, pois é visto que de todas as variáveis que compõem o escore de risco de Framingham, talvez a pressão arterial sistêmica seja a mais intimamente ligada ao estilo de vida do profissional ou trabalhador. Esperavam-se, na presente pesquisa, valores mais altos, pois a tensão no trabalho, imposta nessa categoria, é reconhecidamente elevada. Steptoe ${ }^{12}$ conseguiu demonstrar que tensão no trabalho está associada a respostas de pressão sanguínea aumentada para tarefas ditas não controláveis, ou seja, com baixo poder de decisão, como é o caso dos motoristas (trânsito caótico, insatisfação do passageiro, clima desfavorável).

A hipertensão arterial e o estresse estão relacionados ao desgaste físico e mental, ao cansaço, à má alimentação e ao sedentarismo ${ }^{13}$.

Manter o controle do peso, evitar ingestão excessiva de sal são algumas medidas essenciais. Destaca também que problemas renais podem ter relação com a hipertensão, com a pouca hidratação e com a qualidade da alimentação, pois nem sempre está disponível para o motorista água para consumo que seja de boa qualidade e uma adequada refeição para uma jornada de trabalho cansativa ${ }^{13}$.

Observa-se que, para cada unidade adicional no índice de massa corporal, aumentou em 18,3\% a possibilidade de presença de nível da pressão compatível com hipertensão arterial; para cada aumento da unidade de glicemia $(1 \mathrm{mg} / \mathrm{dL})$ elevou-se em 3,9\% a possibilidade de apresentar hipertensão $\operatorname{arterial~}^{14}$.

A hipertensão arterial apresentou associação significativa com os maiores níveis de índice de massa corporal, circunferência abdominal, glicemia e triglicerídeos, achados já esperados. A hipertensão arterial, a intolerância à glicose, a hipertrigliceridemia, o HDL-c baixo e a hiperinsulinemia, constituem a chamada "Síndrome Metabólica" que promove aumento de risco para doença aterosclerótica e acidente vascular cerebral ${ }^{15}$.

Quanto à história familiar, parentes não apresentaram diabetes $(67,9 \%)$ nem hipertensão (50,9\%), e 96,2\% não possuíam familiares com sobrepeso. Além disso, a quase totalidade dos motoristas negou histórico familiar de patologias cardíacas (Tabela 1).

Há escassez de estudos sobre essa variável na literatura e em profissionais que ocupam outra categoria de trabalho encontraram-se dados divergentes. Do total de 970 funcionários da Petrobrás, entre seus antecedentes familiares, observou-se que para doença arterial coronariana, 25,5\% possuíam história positiva; para hipertensão arterial sistêmica e acidente vascular cerebral, $45,6 \%$ e $12,5 \%$, respectivamente ${ }^{16}$.

Em relação aos dados laboratoriais, grande maioria dos motoristas apresentaram hipertrigliceridemia e hipercolesterolemia. Contudo, os níveis sanguíneos de HDL estavam satisfatórios, bem com o de LDL apresentou nível de normal e desejável em mais da metade da amostra (Tabela 1).

Quanto ao perfil lipídico, mais de um terço apresentou valores de triglicérides $(38,3 \%)$ e colesterol total (33\%) elevados e pouco menos $(25,2 \%)$ para a fração LDL. Além disso, observou-se, percentual muito expressivo $(84 \%)$ de motoristas com HDL abaixo dos níveis considerados desejáveis ${ }^{17}$.

Assim como no presente estudo, no trabalho de Cavagioni et al. ${ }^{17}$ a avaliação do perfil lipídico mostrou que mais de um terço dos motoristas tinha níveis de colesterol nas faixas limítrofe e alta $(33 \%>200 \mathrm{mg} / \mathrm{dL})$ e para o triglicérides $(38 \%$ $>150 \mathrm{mg} / \mathrm{dL}$ ) os dados foram superiores a de outros estudos.

Estes achados não devem ser considerados isoladamente, pois sua expressividade aumenta quando aliados à média de idade da amostra, evidenciando alteração lipídica precoce. Em face dos aspectos discutidos, é inegável a influência dos fatores estruturais, perfil lipídico e glicemia sobre alterações cardiovasculares.

Não houve associação estatisticamente significativa entre IMC e fatores de risco para doença cardiovascular na amostra estudada, sendo $\mathrm{p} \geq$ 0,05. Contudo, é importante destacar a relação inversa entre IMC e nível de estresse e consumo de Coca-Cola, o que não ocorreu com outros tipos de refrigerantes (Tabela 2). 
Tabela 2. Associação entre IMC e fatores de riscos.

\begin{tabular}{|c|c|c|c|c|c|}
\hline Fatores de risco & $\leq 25$ & $>25$ & Total & $\mathrm{OR}(\mathrm{IC} / 95 \%)^{*}$ & Valor-p \\
\hline \multicolumn{6}{|l|}{ Colesterol total } \\
\hline Normal & 6 & 17 & 23 & 1,00 & \multirow[t]{2}{*}{0,817} \\
\hline Elevado & 7 & 23 & 30 & $1,16(0,30-4,08)$ & \\
\hline \multicolumn{6}{|l|}{ Triglicerídeo } \\
\hline Desejável & 4 & 13 & 17 & 1,00 & \\
\hline Elevado & 9 & 27 & 36 & $0,92(0,24-3,56)$ & 0,908 \\
\hline \multicolumn{6}{|l|}{$\mathrm{HDL}$} \\
\hline Baixo/Aceitável & 7 & 31 & 38 & 1,00 & \\
\hline Alto & 6 & 9 & 15 & $0,34(0,09-1,27)$ & 0,100 \\
\hline \multicolumn{6}{|l|}{ LDL } \\
\hline Ótimo/aceitável & 6 & 24 & 30 & 1,00 & \\
\hline Elevado & 7 & 16 & 16 & $0,57(0,16-2,02)$ & 0,382 \\
\hline \multicolumn{6}{|l|}{ VLDL } \\
\hline Normal & 4 & 17 & 21 & 1,00 & \\
\hline Alterado & 9 & 23 & 53 & $0,30(0,16-2,28)$ & 0,453 \\
\hline \multicolumn{6}{|l|}{ Hábito tabagista } \\
\hline Não & 13 & 30 & 43 & & \\
\hline Sim & 0 & 10 & 10 & & \\
\hline \multicolumn{6}{|l|}{ Consumo de bebida } \\
\hline Não & 8 & 21 & 29 & 1,00 & \\
\hline Sim & 5 & 16 & 21 & $1,22(0,34-4,44)$ & \\
\hline \multicolumn{6}{|l|}{ Prática atividade física } \\
\hline Sim & 7 & 17 & 24 & 1,00 & \\
\hline Não & 5 & 19 & 24 & $1,57(0,42-5,86)$ & 0,418 \\
\hline \multicolumn{6}{|l|}{ História familiar de diabetes } \\
\hline Não & 11 & 25 & 36 & 1,00 & \\
\hline Sim & 2 & 15 & 17 & $3,30(0,64-16,91)$ & 0,138 \\
\hline \multicolumn{6}{|l|}{ História familiar de Hipertensão } \\
\hline Não & 8 & 19 & 27 & 1,00 & \\
\hline Sim & 5 & 21 & 26 & $4,77(0,49-6,35)$ & 0,379 \\
\hline \multicolumn{6}{|l|}{ História familiar de obesidade } \\
\hline Não & 13 & 38 & 51 & & \\
\hline $\operatorname{Sim}$ & 0 & 2 & 2 & & \\
\hline \multicolumn{6}{|l|}{ História familiar de insuf.cardíaca } \\
\hline Não & 13 & 36 & 49 & & \\
\hline $\operatorname{Sim}$ & 0 & 4 & 4 & & \\
\hline \multicolumn{6}{|l|}{ História familiar de insuf.cardíaca } \\
\hline Não & 12 & 29 & 41 & 1,00 & \\
\hline \multicolumn{6}{|l|}{ História familiar de A.C.vascular } \\
\hline Sim & 1 & 11 & 12 & $4,55(0,53-39,27)$ & 0,138 \\
\hline História familiar de I.A. miocárdio & & & & & \\
\hline Não & 12 & 36 & 48 & 1,00 & \\
\hline Sim & 1 & 4 & 5 & $1,33(0,14-13,12)$ & 0,805 \\
\hline Consome muito açúcar & & & & & \\
\hline Não & 6 & 12 & 18 & 1,00 & \\
\hline Sim & 7 & 28 & 35 & $2,00(0,55-7,22)$ & 0,285 \\
\hline Consome muita gordura & & & & & \\
\hline Não & 9 & 25 & 34 & 1,00 & \\
\hline $\operatorname{Sim}$ & 4 & 15 & 18 & $1,35(0,35-5,16)$ & 0,660 \\
\hline Consome muito café & & & & & \\
\hline Não & 6 & 10 & 16 & 1,00 & \\
\hline Sim & 7 & 30 & 35 & $2,57(0,70-9,98)$ & 0,149 \\
\hline Consome muita coca-cola & & & & & \\
\hline Não & 6 & 28 & 34 & 1,00 & \\
\hline Sim & 7 & 12 & 19 & $0,37(0,10-1,33)$ & 0,119 \\
\hline Consome muito refrigerante & & & & & \\
\hline Não & 10 & 19 & 29 & 1,00 & \\
\hline Sim & 3 & 21 & 24 & $3,68(0,88-15,42)$ & \\
\hline Consome muito Sal & & & & & \\
\hline Não & 6 & 15 & 21 & 1,00 & \\
\hline Sim & 7 & 25 & 32 & $1,43(0,40-5,06)$ & 0,579 \\
\hline
\end{tabular}

${ }^{\star} \mathrm{OR}=$ Odds Ratio, ${ }^{*} \mathrm{IC} / 95 \%$ = Intervalo de confiança de $95 \%$ 
Praticamente inexiste essa abordagem na literatura em motorista de ônibus urbano, dificultando o embasamento científico do achado.

No trabalho de Rombaldi et al. ${ }^{18}$ envolvendo 972 adultos do município de Pelotas, 20,4\% faziam consumo regular de refrigerante não dietético e $51,3 \%$ apresentavam obesidade, não sendo demonstrado associação importante entre obesidade e consumo de refrigerantes, embora esse estudo não tenha envolvido uma marca específica de bebida açucarada (Coca-Cola).

Uma possível explicação para o aumento de peso decorrente do consumo excessivo de bebidas açucaradas seria o fato de os efeitos fisiológicos da ingestão energética sobre a saciedade serem diferentes para líquidos e para alimentos sólidos. Dessa forma, o carboidrato ingerido em líquidos promoveria um balanço energético positivo maior ${ }^{19}$.

No estudo de Souza et al. ${ }^{20}$ um fator de risco que mostrou associação significativa com CA alterada nos homens foi história familiar precoce de cardiopatia isquêmica, demonstrando a relação existente entre fator genético e ambiente obesogênico (RC = 2,10; 95\% IC:1,17-3,74; p = 0,01).

Houve associação estatisticamente significante entre circunferência abdominal e as seguintes variáveis: história familiar de diabetes $(98,7 \pm 8,5$ vs $91,9 \pm 12,4)$ e de hipertensão $(97,4 \pm 10,3$ vs 90,9 $\pm 12,2$ ) (Tabela 3).

No estudo de Souza et al. ${ }^{20}$, um fator de risco que mostrou associação significativa com CA alterada nos homens foi história familiar precoce de cardiopatia isquêmica, demonstrando a relação existente entre fator genético e ambiente obesogênico (RC = 2,10; 95\% IC:1,17-3,74; p = 0,01).

\section{Conclusão}

No presente estudo, a prevalência para doença cardiovascular mostrou-se baixa, sendo que os principais fatores de risco para doenças cardiovasculares foram: sedentarismo, história familiar positiva e aumento da circunferência abdominal.

Os principais indicadores para doenças cardiovasculares obtidos através dos exames laboratoriais demostraram hipertrigliceridemia e hipercolesterolemia. Contudo, os níveis de HDL estavam satisfatórios, bem como o LDL apresentou nível normal e desejável em mais da metade da amostra.
Tabela 3. Associação entre cintura abdominal e fatores de riscos.

\begin{tabular}{|c|c|c|c|c|}
\hline \multirow[b]{2}{*}{ Fatores de risco } & \multicolumn{2}{|c|}{$\begin{array}{c}\text { Cintura } \\
\text { abdominal }\end{array}$} & \multirow[b]{2}{*}{$t$} & \multirow[b]{2}{*}{ Valor-p } \\
\hline & Média & D.P & & \\
\hline Colesterol total & & & 1,490 & 0,143 \\
\hline Normal & 91,5 & 13,2 & & \\
\hline Elevado & 96,3 & 10,0 & & \\
\hline Triglicerídeo & & & 0,977 & 0,33 \\
\hline Desejável & 91,9 & 10,1 & & \\
\hline Elevado & 95,3 & 12,4 & & \\
\hline HDL & & & 1,888 & 0,065 \\
\hline Baixo/Aceitável & 96,1 & 10,5 & & \\
\hline Alto & 89,5 & 13,4 & & \\
\hline LDL & & & 0,869 & 0,389 \\
\hline Ótimo/aceitável & 92,9 & 12,5 & & \\
\hline Elevado & 95,7 & 10,6 & & \\
\hline VLDL & & & 0,903 & 0,371 \\
\hline Normal & 92,3 & 11,9 & & \\
\hline Alterado & 95,3 & 11,6 & & \\
\hline Hábito tabagista & & & 0,977 & 0,333 \\
\hline Não & 93,4 & 12,5 & & \\
\hline Sim & 97,4 & 6,7 & & \\
\hline Consumo de bebida & & & 0,678 & 0,501 \\
\hline Não & 92,9 & 11,8 & & \\
\hline Sim & 95,2 & 12,2 & & \\
\hline Prática atividade física & & & 0,827 & 0,413 \\
\hline Sim & 92,2 & 11,9 & & \\
\hline Não & 95,0 & 11,4 & & \\
\hline Nível estresse & & & 0,260 & 0,796 \\
\hline Normal & 93,9 & 10,9 & & \\
\hline Alterado & 94,8 & 13,5 & & \\
\hline História familiar de diabetes & & & 2,044 & 0,046 \\
\hline Não & 91,9 & 12,4 & & \\
\hline Sim & 98,7 & 8,5 & & \\
\hline História familiar de Hipertensão & & & & \\
\hline Não & 90,9 & 12,2 & 2,056 & 0,045 \\
\hline Sim & 97,4 & 10,3 & & \\
\hline História familiar de obesidade & & & 0,718 & 0,476 \\
\hline Não & 93,9 & 11,4 & & \\
\hline Sim & 100,0 & 22,6 & & \\
\hline História familiar de A. C. Vascular & & & 0,423 & 0,674 \\
\hline Não & 93,8 & 12,5 & & \\
\hline Sim & 95,4 & 8,8 & & \\
\hline História familiar de I.A. miocárdio & & & 0,209 & 0,836 \\
\hline Não & 94,0 & 11,9 & & \\
\hline Sim & 95,2 & 9,3 & & \\
\hline Consome muito açúcar & & & 1,00 & 0,320 \\
\hline Não & 96,4 & 12,9 & & \\
\hline Sim & 92,9 & 10,9 & & \\
\hline Consome muita gordura & & & 0,812 & 0419 \\
\hline Não & 95,1 & 10,7 & & \\
\hline Sim & 92,3 & 13,5 & & \\
\hline Consome muito café & & & 0,141 & 0,888 \\
\hline Não & 94,5 & 12,7 & & \\
\hline Sim & 94,0 & 11,4 & & \\
\hline Consome muita coca-cola & & & 1,920 & 0,061 \\
\hline Não & 96,4 & 9,9 & & \\
\hline Sim & 90,2 & 13,5 & & \\
\hline Consome muito refrigerante & & & 1,502 & 0,140 \\
\hline Não & 91,9 & 11,3 & & \\
\hline Sim & 96,8 & 11,8 & & \\
\hline Consome muito Sal & & & 1,746 & 0,087 \\
\hline Não & 97,5 & 11,5 & & \\
\hline Sim & 91,9 & 11,4 & & \\
\hline
\end{tabular}

${ }^{\star}$ D.P $=$ Desvio padrão; $\mathrm{t}=$ Teste $\mathrm{t}$ de "Student". 


\section{Colaboradores}

AF Alquimim, ABCR Barral, KC Gomes e MC Rezende participaram igualmente de todas as etapas de elaboração do artigo.

\section{Referências}

1. Alves CS, Paula PS. Violência no trabalho: possíveis relações entre assaltos e TEPT em rodoviários de uma empresa de transporte coletivo. Cad psicol soc Trab 2009; 12(1):35-46.

2. Pinto FM, Neves MY. A gestão da atividade do motorista de ônibus: um olhar ergológico. Estud pesq Psicol 2009; 9(2):493-511.

3. Battiston M, Cruz RM, Hoffman MH. Condições de trabalho e saúde de motoristas de transporte urbano coletivo. Estud de psicol 2006; 11(Supl. 1):333-343.

4. Baltazar CC, Maia ER, Figueiredo FJS, Correia BRB, Holanda SA. Motorista de ônibus urbano e intermunicipal do triângulo de Crajubar: uma abordagem ergonômica. Saude Colet 2008; 9(2). [site na Internet]. [acessado 2012 jul 11]. Disponível em: http://coletanea2008.no.comunidades.net/index. php?pagina $=1225359146$

5. Neri M, Soares C. Condições de saúde no setor de transporte de carga e passageiros. Cad Saude Publica 2005; 21(4):1107-1123.

6. Facirolli K. A influência do exercício físico sobre respostas cardiorrespiratórias na obesidade grau III: um relato de caso. Rev ENAF Science 2009; 4(2):1518.

7. Landim MBP, Guimarães E. Escore de Framingham em motoristas de transportes coletivos urbanos de Teresina, Piauí. Arq Bras Card 2006; 87(3):315-320.

8. Rocha VC, Carvalho ZM, Araújo MFM, Marques MB. Fatores de risco para acidente vascular encefálico em motoristas de ônibus urbano de Fortaleza-CE. In: Anais do VI Congresso Internacional de Cardiologia; 2009; Fortaleza (CE). p. 1-3.

9. Costa LB, Koyama MAH, Minuci EG, Fischer FM. Morbidade declarada e condições de trabalho: o caso dos motoristas de São Paulo e Belo Horizonte. Rev São Paulo em Perspectiva 2003; 17(2):54-67.

10. Faria BK, Amorim G, Vancea DMM. Perfil alimentar e antropométrico dos motoristas da empresa de transporte coletivo JOTUR/Palhoça-SC. Rev Bras Obesidade Nutrição e Emagrecimento 2007; 1(1):11-20.

11. Lima SCVC, Arrais RF, Pedrosa LFC. Avaliação da dieta habitual de crianças e adolescentes com sobrepeso e obesidade. Rev Nutr 2004; 17(4):469-477.

12. Steptoe A. Job strain, blood pressure and response to uncontrollable stress. J Hypertens 1999; 17(2):193200.
13. Soares TC. Percepção de motoristas de ônibus intermunicipal sobre os riscos ocupacionais que possam afetar sua saúde [monografia]. Mato Grosso: Faculdade de Arquitetura, Engenharia e Tecnologia; 2005.

14. Cavagioni LC, Pierin AMG. Hipertensão arterial e obesidade em motoristas profissionais de transporte de cargas. Acta Paul Enferm 2010; 23(4):455-460.

15. Ozonato E, Ramos SP. O estresse na profissão de motorista de transporte coletivo urbano por ônibus. Mato Grosso do Sul: UFMS; 2001.

16. Matos MFD, Souza e Silva NA, Pimenta AJM, Cunha AJLA. Prevalência dos fatores de risco para doença cardiovascular em funcionários do Centro de Pesquisas da Petrobras. Arq Bras Cardiol 2004; 82(1):1-8.

17. Cavagioni LC, Bensenor IM, Halpern A, Pierin AMG. Síndrome metabólica em motoristas profissionais de transporte de cargas da rodovia BR-116 no trecho paulista Régis Bittencourt. Arq Bras Endocrinol Metab 2008; 56(6):1015-1023.

18. Rombaldi AJ, Neutzling MB, Silva MC, Azevedo MR, Hallal PC. Fatores associados ao consumo de refrigerante não dietético em adultos de pelotas, RS. Rev Saude Publ 2011; 45(2):382-390.

19. Botolli C, Moraes MA, Goldemeir S. Fatores de risco cardiovasculares em trabalhadores de enfermagem de um centro de referência do sul do Brasil. Ciencia y Enfermeria Concepcion 2009; 15(3):101-109.

20. Souza PAL, Fayh APT, Portal VL. Circunferência abdominal como preditor de evolução em 30 dias na síndrome coronariana aguda. Arq Bras Card 2011 [acessado 2012 jul 11];96(5): [cerca de 6 p.]. Disponível em: http://www.scielo.br/pdf/abc/2011nahead /aop03211.pdf

Artigo apresentado em 10/05/2011

Aprovado em 03/06/2011

Versão final apresentada em 02/09/2011 